\title{
Rethinking the need for overnight admission after peroral endoscopic myotomy (POEM): a pandemic-driven approach to the future
}

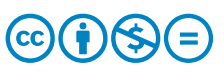

\author{
Authors \\ Linda Y. Zhang, Michael Bejjani, Bachir Ghandour, Mouen A. Khashab
}

Institution

Johns Hopkins Medical Institutions, Baltimore, Maryland,

United States

submitted 5.2.2021

accepted after revision 31.3.2021

Bibliography

Endosc Int Open 2021; 09: E1381-E1385

DOI 10.1055/a-1490-9385

ISSN 2364-3722

(c) 2021. The Author(s).

This is an open access article published by Thieme under the terms of the Creative Commons Attribution-NonDerivative-NonCommercial License, permitting copying and reproduction so long as the original work is given appropriate credit. Contents may not be used for commercial purposes, or adapted, remixed, transformed or built upon. (https://creativecommons.org/licenses/by-nc-nd/4.0/)

Georg Thieme Verlag KG, Rüdigerstraße 14,

70469 Stuttgart, Germany

Corresponding author

Mouen A. Khashab, MD, Associate Professor of Medicine,

Director of Therapeutic Endoscopy, Division of

Gastroenterology and Hepatology, Johns Hopkins Medical

Institutions, United States

Fax: +1-410-292-2077

mkhasha1@jhmi.edu

\section{ABSTRACT}

Background and study aims COVID-19 has significantly impacted endoscopic assessment and management of multiple conditions. Our group recommenced treatment of highly symptomatic achalasia patients (Eckardt score $\geq 6$ ) adopting a same-day discharge (SDD) algorithm and present early outcomes of its utilization.

Patients and methods We enrolled all outpatients undergoing POEM at a single tertiary referral center. Patients qualified for SDD if all of the following a priori criteria were met: 1) ASA grade I-III; 2) No intraprocedural adverse events (AEs); 3) Secure mucosal closure; 4) Post-procedure pain/ nausea responsive to oral medications; and 5) Patients tolerating clear fluids.

Results In 17 potential SDD candidates (female $82.4 \%$, median age 51 years [IQR 48-64]) undergoing POEM, SDD was achieved in 14 (82.4\%). The remaining were admitted due to post-procedure pain/nausea $(n=2)$ and intraprocedural concerns (capnoperitoneum requiring needle decompression, $n=1$ ). There were no post-procedure AEs or unexpected readmissions.

Conclusions This study demonstrates the safety and feasibility of a SDD algorithm for POEM patients and challenges the necessity of routine post-POEM hospital admission. Critically important during the COVID-19 pandemic, this may in fact constitute a changing standard for POEM post-procedure care.

\section{Introduction}

The current COVID-19 pandemic has had a profound impact on endoscopy units worldwide, with most units at some point restricting to urgent and life-saving procedures as a means of minimizing infection spread and diverting health care resources to the front line. However, 1 year after the World Health Organization declared a public health emergency due to the outbreak of a new coronavirus, consideration also needs to be given to those various gastrointestinal conditions whose diagnosis and treatment were delayed.
Achalasia is an esophageal motility disorder resulting in high morbidity due to dysphagia, regurgitation, chest pain and/or weight loss. Peroral endoscopy myotomy (POEM) has rapidly emerged as a first-line treatment option, carrying an overall severe adverse event $(A E)$ rate of $<0.5 \%$ [1]. Despite its widespread uptake, the POEM procedure is yet to be standardized and there is no clear agreement regarding post-procedure care. A common practice involves a 24-hour observation and esophagogram prior to commencing diet and discharging the patient. This is likely due to high morbidity and mortality in the event of mediastinitis from esophageal leak; however, the true incidence of this post-POEM is low at $0.7 \%$ [1]. 


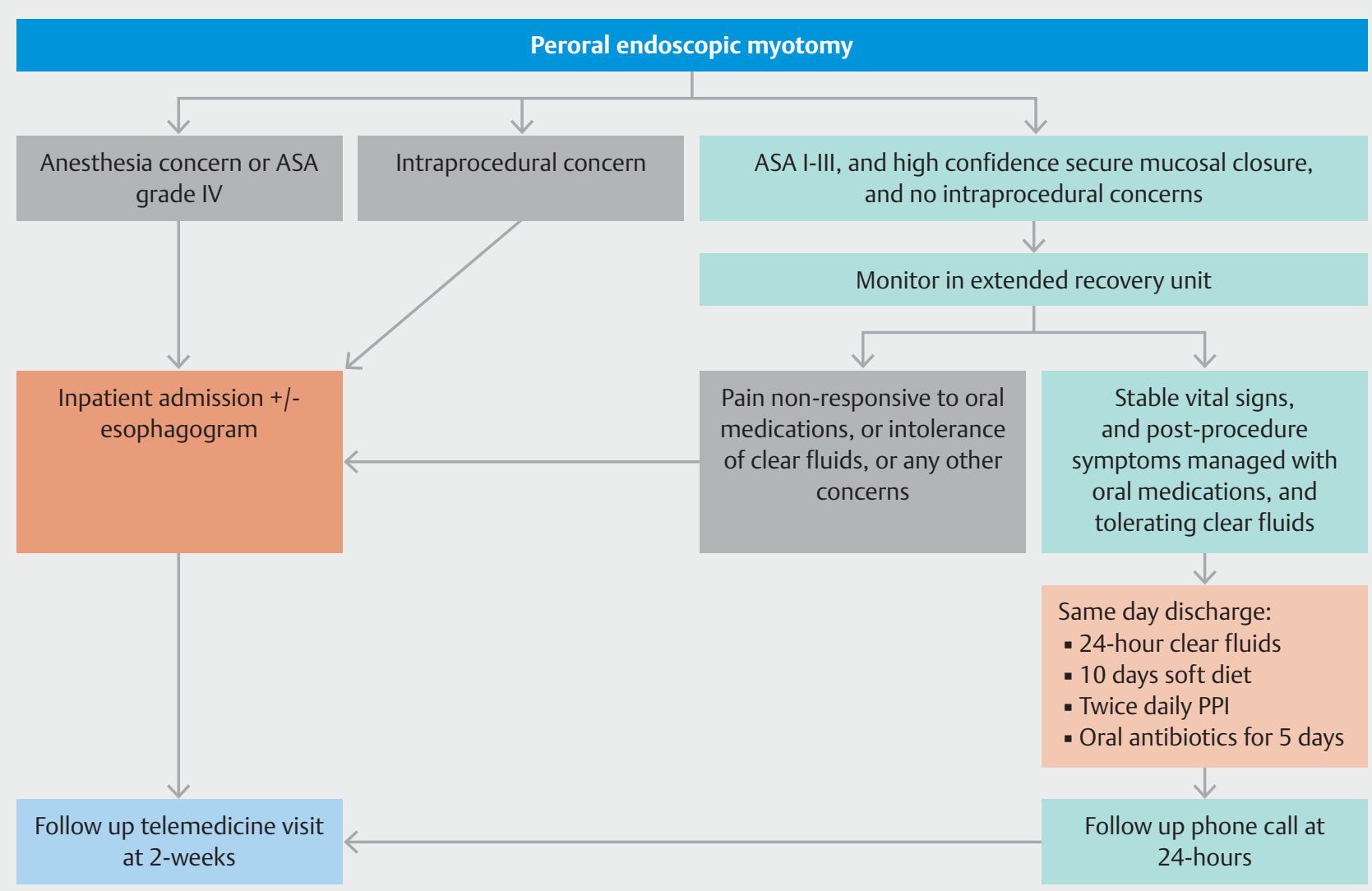

- Fig. 1 Peroral endoscopic myotomy (POEM) same-day discharge algorithm.

ASA, American Society of Anesthesiologists physical status classification; PPI, proton pump inhibitor.

Limited early data have suggested that same-day discharge (SDD) can be safely and feasibly performed [2-4]. This is yet to be widely implemented but of particular importance now as COVID-19 continues to place profound burden on healthcare resources. The number of COVID patients in the United States continues to rise and at the time of writing hospital bed occupancy at our institution has consistently exceeded $90 \%$ for the past few weeks despite extensive ward conversions. Minimizing unnecessary utilization of inpatient hospital resources is crucial now more than ever. Our group recommenced treatment of highly symptomatic patients with achalasia (Eckardt score $[E S] \geq 6$ ) and adopted an SDD algorithm. We present early outcomes of its utilization.

\section{Patients and methods}

\section{Study design}

Consecutive outpatients who presented for POEM and were considered possible SDD candidates between November 17, 2020 and January 8, 2021 at a single tertiary referral center were included. Patients qualified for SDD if all of the following a priori criteria were met ( $\mathbf{F i g . 1 ) : ~ 1 ) ~ A m e r i c a n ~ S o c i e t y ~ o f ~ A n - ~}$ esthesiologists (ASA) physical status classification system grade I-III; 2) No intraprocedural complications; 3) High confidence for a secure mucosal closure; 4) Post-procedure pain and nausea were responsive to oral medications; and 5) Patients tolerating clear fluids. Patients not meeting these criteria, or with any other concerns, were admitted to the inpatient ward.

\section{POEM procedure}

All POEMs were performed in an endoscopy unit with the patient under general anesthesia and in a supine position. Distensibility measurements at the gastroesophageal junction (GEJ) using the functional lumen imaging probe was performed preand post-POEM (prior to mucosal closure). Intraprocedural antibiotics (cefotetan) were given prior to incision. Anterior approach was favored unless circumstances dictated otherwise such as in the case of prior treatment and/or expected scarring. Mucosal incision was performed 10 to $15 \mathrm{~cm}$ proximal to the $G E J$. The submucosal tunnel extended for $3 \mathrm{~cm}$ beyond the lower esophageal sphincter (LES) into the gastric cardia. A selective circular myotomy was performed within the esophagus (ending $3 \mathrm{~cm}$ distal to the mucosal incision), while a full thickness myotomy was performed across the LES and gastric muscle. Finally, the mucosa was closed with through the scope clips (TTSC) and careful inspection was performed to ensure adequate closure and lack of inadvertent injury. We defined high confidence secure mucosal closure as the sequential and symmetric placement of TTSC across the mucosal defect, with each clip in close 
proximity to the previous and adequate mucosal apposition (equal amount of tissue grasped on either side of the defect, creating a symmetric fold after clip deployment). Incorrectly positioned clips were removed before placement of subsequent clips. In case of difficult closure, suboptimal clip positioning or where visualization of the completed mucosal closure was impeded, the closure was considered not confidently closed, in which case, the patient would be admitted.

\section{Post-procedure care}

Patients were observed for a minimum of 4 hours. Patients meeting SDD criteria were provided with clear written instructions, acetaminophen and tramadol as required for pain, promethazine or ondansetron as required for nausea, a 5-day course of oral antibiotics (amoxicillin/clavulanic acid), and a 4week course of twice-daily proton pump inhibitor therapy. The discharge instructions included contact details for 24-hour access to an on-call gastroenterology provider, who could then communicate directly with the proceduralist if required. Patients were also able to message their treating team through our electronic patient portal. As part of routine care, patients were advised to seek medical attention immediately in the event of severe sore throat, severe abdominal discomfort, severe nausea/vomiting or hematemesis, fever, chest pain or shortness of breath. Patients were allowed clear fluids on the day of procedure and if they were well at a telephone followup on post-procedure day one, advanced to a soft diet for 10 days. Patients were then reviewed at a telemedicine visit at two-weeks.

\section{Data collection and statistical analysis}

Data were prospectively collected and included baseline clinical characteristics, pre- and post-POEM Eckardt scores, procedural characteristics, discharge and admission rates, length of hospital stay, and any AEs. Categorical variables are reported as numerical counts and percentages. Continuous data are reported as median and interquartile range (IQR).

\section{Results}

During the study period, 17 patients (female $82.4 \%$, median age 51 years [48-64] and ASA 2 [2-3]) who were candidates for SDD were treated with POEM ( $>$ Table 1 ). The median prePOEM Eckardt score was $6(6-8)$ and the majority of patients $(88.2 \%)$ were treatment naïve.

All POEMs were technically successful with a median procedural time of 42 minutes (37-49) ( $\downarrow$ Table 2$)$. All but one patient underwent an anterior approach. The median length of esophageal myotomy was $7 \mathrm{~cm}$ (7-10) and all gastric myotomies were $3 \mathrm{~cm}$ long. One patient who had combined POEM and diverticulotomy had capnoperitoneum during the procedure requiring needle decompression. No other intraprocedural AEs, including inadvertent mucosal injuries, were noted. All mucosal incisions were closed with high confidence using a median of six clips (5-6). Median distensibility index improved from $0.7 \mathrm{~mm}^{2} / \mathrm{mmHg}$ pre-POEM $(0.5-1)$ to $4.9 \mathrm{~mm}^{2} / \mathrm{mmHg}$ post-POEM (4.2-5.6).
- Table 1 Baseline characteristics.

\begin{tabular}{|l|c|}
\hline Clinical characteristic & $51(48-64)$ \\
\hline Age, median (IQR) & $14(82.4 \%)$ \\
\hline Female, $\mathrm{n}(\%)$ & \\
\hline Manometric subtype, $\mathrm{n}(\%)$ & $1(5.9 \%)$ \\
\hline - Achalasia type I & $8(47.1 \%)$ \\
\hline - Achalasia type II & $3(17.6 \%)$ \\
\hline - Achalasia type III & $4(23.5 \%)$ \\
\hline - Jackhammer esophagus & $1(5.9 \%)$ \\
\hline - Esophagogastric junction outlet obstruction & $2(11.8 \%)$ \\
\hline Previous achalasia treatment, n (\%) & $6(6-8)$ \\
\hline Baseline Eckhardt score, median (IQR) & \\
\hline ASA score & $0(0 \%)$ \\
\hline - I & $10(58.8 \%)$ \\
\hline - II & $7(41.2 \%)$ \\
\hline - III & $0(0 \%)$ \\
\hline - IV & \\
\hline
\end{tabular}

IQR, interquartile range; ASA, American Society of Anesthesiologists physical status classification.

SDD was achieved in 14 patients (82.4\%), with the remaining being admitted due to post-procedure pain or nausea $(n=$ 2 ) and intraprocedural concerns in the aforementioned patient with capnoperitoneum $(n=1)$. All SDD patients were well at last follow up and experienced no post-discharge AEs. There were no unexpected readmissions. In patients at least 2 weeks postPOEM ( $n=14$, median follow up 21 (17-24) days), all were tolerating a solid diet and median ES improved to 0 (0-1).

\section{Discussion}

The ongoing COVID-19 pandemic has had a drastic impact on endoscopy units worldwide, the effects of which are likely to outlast the pandemic. Our trigger for this case series was twofold. The absolute priority was to avoid diversion of frontline resources away from the pandemic. Second, we hoped to resume treatment for highly symptomatic patients whose therapy had been significantly delayed while preserving inpatient beds, minimizing healthcare costs and decreasing the risk of nosocomial infections. To this end we proposed a POEM SDD algorithm ( Fig. 1) to treat highly symptomatic achalasia patients with Eckardt score $\geq 6$ and demonstrated that it is safe and feasible. This is a deviation from standard practice at many institutions where patients are admitted overnight and undergo routine esophagogram. Using our algorithm, we were able to discharge 14 of 17 patients (82.4\%). Of the remaining three, two were admitted due to postoperative pain/nausea. The final patient had a prior surgical diverticulectomy complicated by leak requiring repair but developed recurrent lower esophageal diverticulum 


\begin{tabular}{|c|c|}
\hline \multicolumn{2}{|l|}{ Procedural outcomes } \\
\hline Procedural time, mins, median (IQR) & $42(37-49)$ \\
\hline \multicolumn{2}{|l|}{ Approach, n (\%) } \\
\hline - Anterior & $16(94.1 \%)$ \\
\hline - Posterior & $1(5.9 \%)$ \\
\hline Tunnel length, median (IQR), cm & $13(13-14)$ \\
\hline \multicolumn{2}{|l|}{ Myotomy length, median (IQR), cm } \\
\hline - Esophageal & $7(7-10)$ \\
\hline - Gastric & $3(3-3)$ \\
\hline Through the scope clips for closure, median (IQR) & $6(5-6)$ \\
\hline $\begin{array}{l}\text { Preoperative distensibility index, median (IQR), } \\
\mathrm{mm}^{2} / \mathrm{mmHg}\end{array}$ & $0.7(0.5-1)$ \\
\hline $\begin{array}{l}\text { Postoperative distensibility index, median (IQR), } \\
\mathrm{mm}^{2} / \mathrm{mmHg}\end{array}$ & $4.9(4.2-5.6)$ \\
\hline \multicolumn{2}{|l|}{ Clinical outcomes } \\
\hline Same day discharge, $\mathrm{n}(\%)$ & $14(82.4 \%)$ \\
\hline Patients requiring admission, $\mathrm{n}(\%)$ & $3(17.6 \%)$ \\
\hline - Post-procedure pain or nausea & 2 \\
\hline - Intraprocedural concern & 1 \\
\hline $\begin{array}{l}\text { Length of stay post procedure, median (IQR), } \\
\text { hours }\end{array}$ & $5(4.5-6.5)$ \\
\hline - Sam day discharge patients & $4.9(4.5-5.2)$ \\
\hline - Admitted patients & $34(31.6-39.4)$ \\
\hline Adverse events & $1(5.9 \%)$ \\
\hline Two-week Eckardt score, median (IQR) $(n=14)$ & $0(0-1)$ \\
\hline Longest follow-up (days), median (IQR), days & $21(17-24)$ \\
\hline
\end{tabular}

due to previously undiagnosed achalasia. He had a combined POEM and diverticular septotomy, resulting in a longer total procedure time. Further, his procedure was complicated by the presence of surgical clips, suture material, and significant scarring. The patient had intraprocedural capnoperitoneum requiring needle decompression but recovered well and was discharged post-procedure day 1. Importantly, all patients who had SDD remained well and had no unexpected readmissions relating to POEM. We attribute our success in this regard to close follow-up of patients (with telephone call on day 1 and telemedicine visit at 2 weeks) and the ability for patients to contact the treating team through a messaging system on our electronic patient portal.

Meticulous patient selection, procedural technique, and implementation of safety nets are crucial for implementation of any new SDD protocol. Despite the COVID-19 pandemic calling for frequent and rapid changes, new protocols should never compromise patient safety. Criteria should take into considera- tion baseline clinical characteristics, procedural characteristics, and early post-procedure recovery progress. As international POEM experience grows, endoscopists are learning intraprocedural features associated with post-procedure complications. Our definition of a high-confidence, secure mucosal closure was based on assessment by an expert endoscopist. Although the ability to identify and rectify small technical issues during closure comes with experience, certain factors have been associated with AEs. Asymmetric closure can result in pocket formation, leading to submucosal tunnel infection and rupture of the incision site [5]. We perform mucosal closure from distal to proximal, ensuring the first clip is placed just at or slightly beyond the distal margin of the mucosal incision. Optimal positioning of the first clip is vital, as this can significantly impact the technical difficulty of subsequent clips. The state of the mucosa around the incision site should be considered. For example, suturing could be considered in the setting of thick mucosa, chronic esophagitis with compromised integrity, or significant coagulation at the edges [6]. Limited literature has shown that longer procedure duration is associated with increased likelihood for admission [3,4]. Development of postprocedure pain or nausea is more difficult to predict, and detection relies on extended monitoring in the recovery unit. A routine part of post-procedure care at many centers, the role of post-POEM esophagogram has been questioned after several studies reported frequent findings (such as pneumoperitoneum) of no clinical significance while occasional serious AEs were missed $[7,8]$. Further, day 1 esophagograms do not predict longer-term outcomes [9] but can contribute to delayed discharge [3]. Given these reasons in addition to the overall low rate of esophageal leak at $0.7 \%$ [1], routine esophagogram may be an unnecessary use of our limited healthcare resources, particularly during the COVID-19 pandemic. We propose that at expert centers, proceduralist confidence in secure mucosal closure and the lack of other concerning intraprocedural complications can take the place of routine esophagograms.

Our study does have a few limitations. First, it had a small cohort from a single expert center. Our center has a high volume of achalasia patients leading to significant experience among the entire treating team; however, this may not be applicable to all centers. Second, our follow-up duration was short, but we feel that this is adequate to demonstrate the safety and feasibility of our algorithm. Finally, larger-scale studies will be needed to prospectively identify clinical, procedural, and technical factors that predict need for admission.

\section{Conclusions}

This study challenges the necessity of routine post-POEM hospital admission and esophagograms. Although routine hospital admission and esophagogram will continue to be of merit in those early on in their POEM practice or lacking the support of an experienced team (including but not limited to anesthesia and nursing), SDD following POEM could certainly be considered in expert centers. Borne out of necessity during the COVID-19 pandemic in which minimizing unnecessary utilization of inpatient hospital resources is an absolute priority, this may in 
fact constitute a changing standard for POEM post-procedure care, lasting even beyond the pandemic.

\section{Competing interests}

Dr. Khashab is a consultant for Boston Scientific, Olympus America, Medtronic and GI Supply.

\section{References}

[1] Haito-Chavez Y, Inoue H, Beard KW et al. Comprehensive analysis of adverse events associated with per oral endoscopic myotomy in 1826 patients: an international multicenter study. Am J Gastroenterol 2017; 112: 1267-1276

[2] Cloutier Z, Mann A, Doumouras AG et al. Same-day discharge is safe and feasible following POEM surgery for esophageal motility disorders. Surg Endosc 2020: doi:10.1007/s00464-020-07781-4

[3] Attaar M, Su B, Wong HJ et al. Factors associated with admission after implementation of a same-day discharge pathway in patients undergoing peroral endoscopic myotomy (POEM). Surg Endosc 2020: doi:10.1007/s00464-020-07866-0
[4] Benias PC, Korrapati P, Raphael KL et al. Safety and feasibility of performing peroral endoscopic myotomy as an outpatient procedure with same-day discharge. Gastrointest Endosc 2019; 90: 570-578

[5] Li QL, Yao LQ, Xu XY et al. Repeat peroral endoscopic myotomy: a salvage option for persistent/recurrent symptoms. Endoscopy 2016; 48 : 134-140

[6] Khashab MA, Sethi A, Rosch T et al. How to perform a high-quality peroral endoscopic myotomy? Gastroenterology 2019; 157: 11841189

[7] Reddy CA, Tavakkoli A, Abdul-Hussein M et al. Clinical impact of routine esophagram after peroral endoscopic myotomy. Gastrointest Endosc 2021; 93: 102-106

[8] El Khoury R, Teitelbaum EN, Sternbach JM et al. Evaluation of the need for routine esophagram after peroral endoscopic myotomy (POEM). Surg Endosc 2016; 30: 2969-2974

[9] Sternbach JM, El Khoury R, Teitelbaum EN et al. Early esophagram in per-oral endoscopic myotomy (POEM) for achalasia does not predict long-term outcomes. Surgery 2015; 158: 1128-1135 